\title{
Comparison of the Measured Electrical Properties of Pig Internal Organs with the Given Values for Human Organs
}

\author{
Ji-Hyun Jung $\cdot$ Se-Yun Kim
}

\begin{abstract}
The electrical properties of pig internal organs including lung, liver, heart, kidney, blood, stomach, and small intestine are measured using an open-ended coaxial probe and an improved virtual transmission-line model. The measured complex permittivities of the pig organs are compared with the given values of the corresponding human organs. A similarity between these values is confirmed. For organs such as lung, liver, heart, and kidney that have regular texture and contents, the complex permittivities are almost identical to those of the corresponding human organs. The complex permittivities of human and pig blood are also very close in value. However, relatively large deviations are observed for the cases of stomach and small intestine because the internal contents of these organs significantly affect the measured electrical properties.
\end{abstract}

Key words: Electrical Property, Open-Ended Coaxial Probe, Pig Internal Organ.

\section{Introduction}

Electromagnetic fields radiated from implanted or ingested sources through the complex human body have been widely analyzed by many research groups for the development of wireless biomedical devices [1] [3]. For the same purpose, dominant propagation path and corresponding phenomenon from intestine ingested source was numerically analyzed in our group [4], [5]. Our next goal is experimental confirmation of the numerically analyzed results. However, direct measurement with human body is impossible due to the safety problem. Therefore, preparation of a precise phantom is important for obtaining accurate experimental results.

Several internal organs of animals are being considered by our group to organize more realistic phantom. The internal organs from pig are especially considered due to the known similarity of electrical properties between human and pig organs [6]. As the first step of preparation, complex permittivities of several pig internal organs are measured to check the similarity between their values. Dielectric properties of the employed pig organs also need to beknown for precise control of the overall experimental process.

Our open-ended coaxial probe and the improved vir- tual transmission-line model are employed during the conversion process [7]. Electrical properties of pig lung, liver, heart, kidney, blood, stomach, and small intestine are obtained. These measured complex permittivities are then compared with the corresponding values of human organs given by Gabriel [8].

The preparation of pig internal organs and experimental configuration employing the open-ended coaxial probe and the improved virtual transmission-line model are described in Section II. Analysis of the measured results is presented in Section III. Finally, conclusions are summarized in the last Section.

\section{II . Preparation of Experimentation}

The overall experiment is performed at as laughterhouse located in Doksan-dong, Seoul, Korea. This allows complex permittivities of the pig internal organs to be measured immediately after slaughter, without degradation of the organs. For the same reason, our openended coaxial probe, shown in Fig. 1, is employed since direct measurement over a wide range of frequencies is possible without any other processing. The inner and outer conductors of the open-ended coaxial probe have radii of $0.635 \mathrm{~mm}$ and $2.04 \mathrm{~mm}$, respectively, and are

Manuscript received March 7, 2012 ; Revised April 12, 2012 ; Accepted May 7, 2012. (ID No. 20120307-006J)

Korea Institute of Science and Technology, Seoul, Korea.

Corresponding Author : Se-Yun Kim (e-mail : ksy@imrc.kist.re.kr)

This is an Open-Access article distributed under the terms of the Creative Commons Attribution Non-Commercial License (http:/creativecommons.org/licenses/ by-nc/3.0) which permits unrestricted non-commercial use, distribution, and reproduction in any medium, provided the original work is properly cited. 


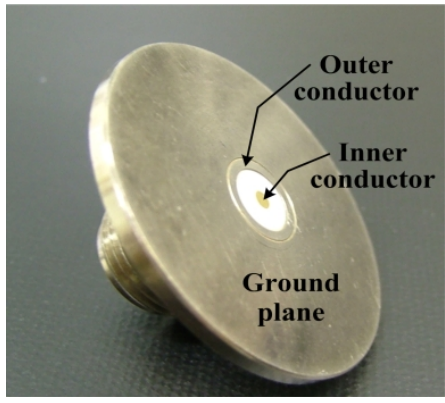

Fig. 1. Geometry of the open-ended coaxial probe used for measurements.

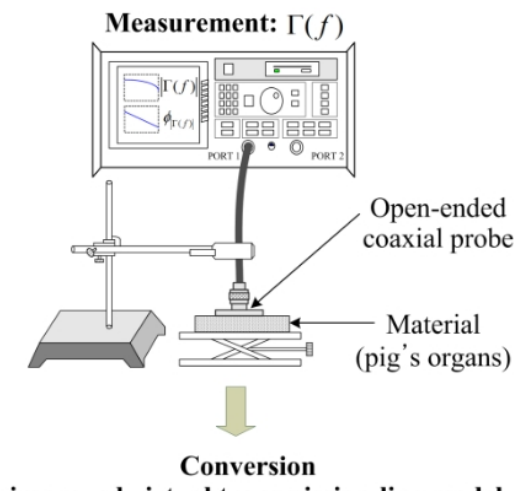

: improved virtual transmission-line model

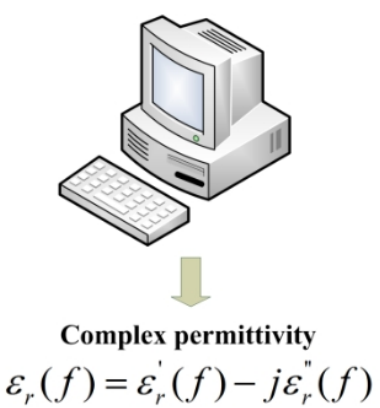

Fig. 2. Experimental procedure for measuring complex permittivities of pig internal organs.

separated by a Teflon filling. The radius of the ground plane is $12 \mathrm{~mm}$.

The prepared internal organs are lung, liver, heart, kidney, blood, stomach, and small intestine. As illustrated in Fig. 2, the cutting plane of the open-ended coaxial probe is contacted with the freshly excised organs. The other side of the probe is connected to the vector network analyzer through N-type coaxial cable. A portable network analyzer, Insite 2.0, is utilized for the measurements. The reflection coefficients $(\Gamma)$ are measured at the contact plane and inserted into a conversion model, the improved virtual transmission-line model developed by our group [7]. As a result, the complex permittivities $\left(\epsilon_{r}\right)$ of the pig organs are converted to a frequency range of $15.6 \mathrm{MHz}$ to $2.5 \mathrm{GHz}$.
Table 1. Given dielectric properties of some human organs characterized by the 1-pole Debye formula.

\begin{tabular}{|c|c|c|c|c|}
\hline & $\epsilon_{r s}$ & $\epsilon_{r \infty}$ & $\tau\left(\times 10^{-10}\right)$ & $\sigma$ \\
\hline Lung & 28.9378 & 21.0586 & 5.2001 & 0.31 \\
\hline Liver & 63.0376 & 44.4947 & 5.0439 & 0.49 \\
\hline Heart & 82.6913 & 56.6345 & 5.0521 & 0.55 \\
\hline Kidney & 87.7882 & 54.7103 & 5.1588 & 0.79 \\
\hline Blood & 72.1037 & 59.9207 & 5.1822 & 1.25 \\
\hline Stomach & 73.7655 & 63.6256 & 4.8196 & 0.91 \\
\hline $\begin{array}{c}\text { Small } \\
\text { intestine }\end{array}$ & 86.6136 & 56.4308 & 5.5638 & 1.63 \\
\hline
\end{tabular}

\section{Analysis of the Measured Dielectric Properties}

The converted dielectric properties of the pig internal organs are compared with the corresponding values for human organs at frequency band from $100 \mathrm{MHz}$ to 700 $\mathrm{MHz}$ [4], [5]. The complex permittivities of human organs are obtained from Gabriel et al. [6]. The frequency-dependent characteristics of human organs are expressed by the 1-pole Debye formula [7],

$$
\epsilon_{r}(f)=\left(\epsilon_{r \infty}+\frac{\epsilon_{r s}-\epsilon_{r \infty}}{1+j 2 \pi f \tau}\right)+\frac{\sigma}{j 2 \pi f \epsilon_{0}},
$$

where $\epsilon_{r s}$ is the zero frequency permittivity, $\epsilon_{r \infty}$ is the high frequency permittivity, $\epsilon_{0}$ is the free space permittivity, $\sigma(S / m)$ is the conductivity, and $\tau(s)$ is the relaxation time. The parameters of the employed organs are listed in Table 1.

The real and imaginary parts of the measured complex permittivities of lung, liver, heart, and kidney are similar to the corresponding values of human organs, as shown in Figs. 3, 4, 5, and 6, respectively. The elec-

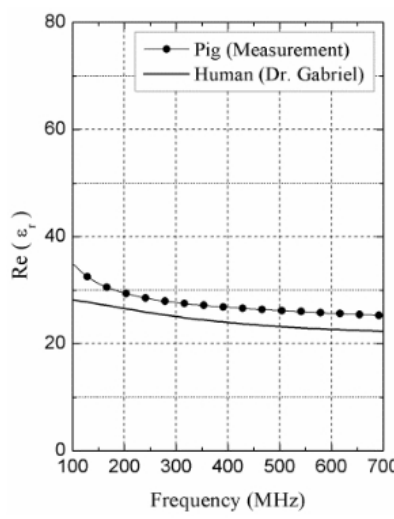

(a) Real part

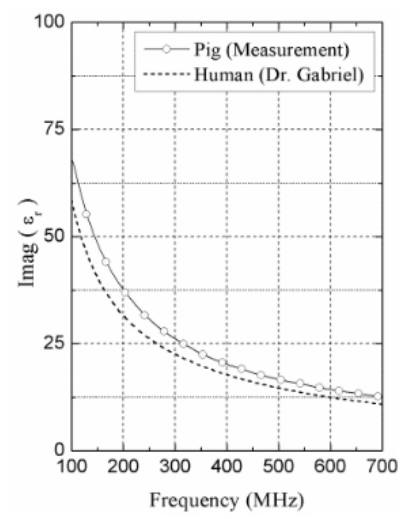

(b) Imaginary part
Fig. 3. Measured electrical property of pig lung compared with the corresponding value from human lung. 


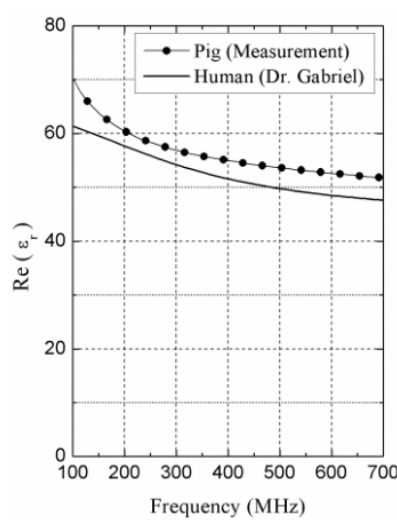

(a) Real part

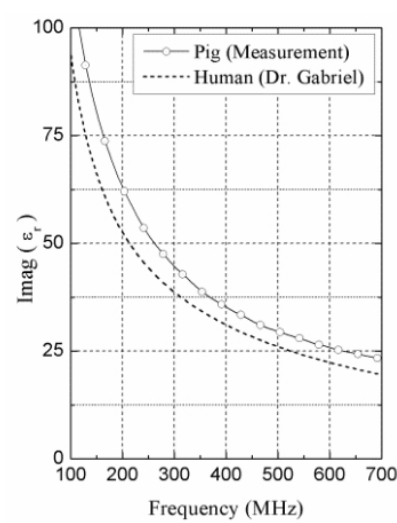

(b) Imaginary part
Fig. 4. Measured electrical property of pig liver compared with the corresponding value from human liver.

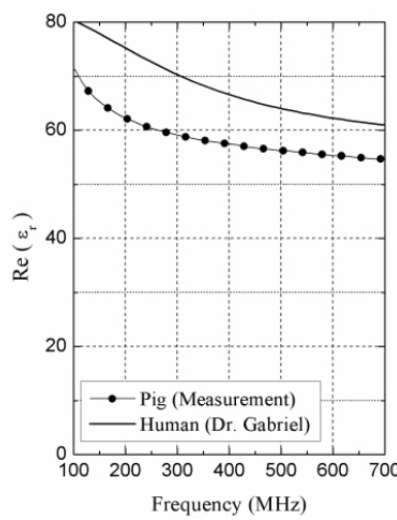

(a) Real part

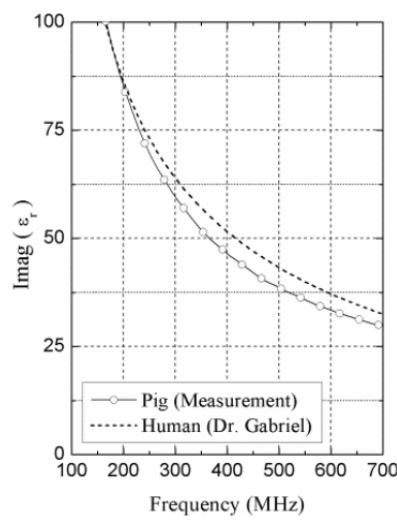

(b) Imaginary part
Fig. 5. Measured electrical property of pig heart compared with the corresponding value from human heart.

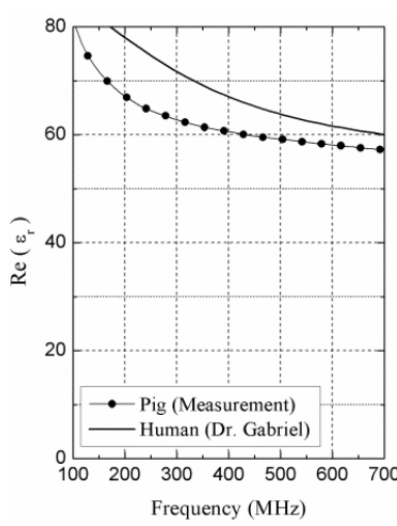

(a) Real part

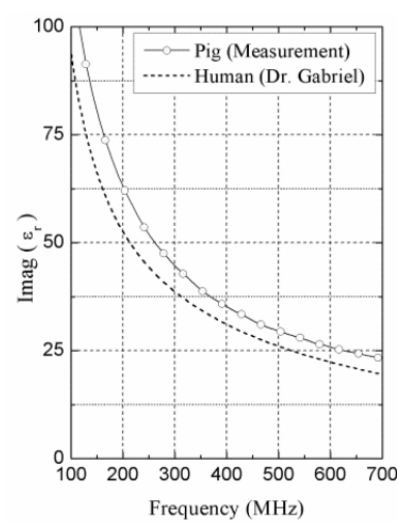

(b) Imaginary part
Fig. 6. Measured electrical property of pig kidney compared with the corresponding value from human kidney.

trical property of pig blood, shown in Fig. 7, is also very close to the value for human blood. However, relatively large deviations are observed for the results from

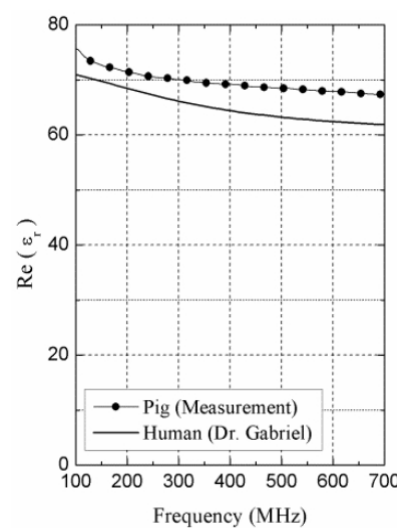

(a) Real part

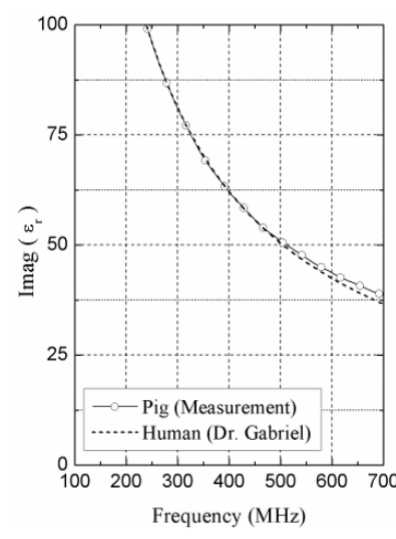

(b) Imaginary part
Fig. 7. Measured electrical property of pig blood compared with the corresponding value from human blood.

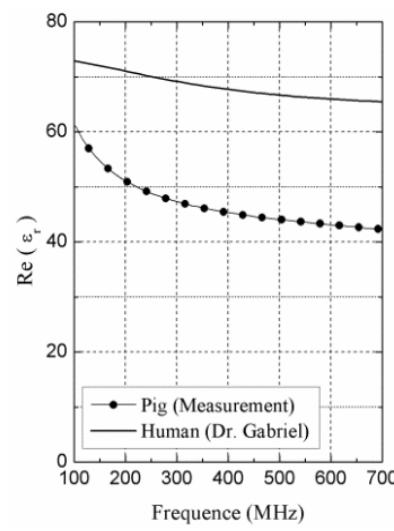

(a) Real part

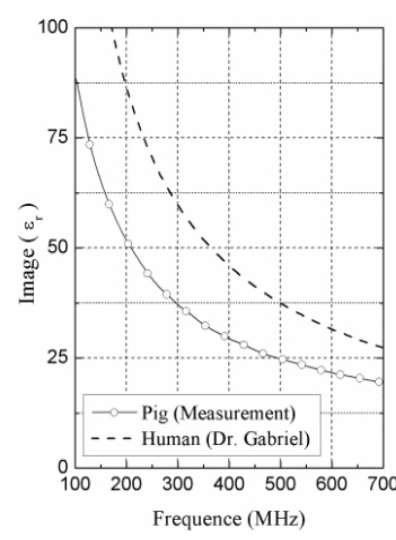

(b) Imaginary part
Fig. 8. Measured electrical property of pig stomach compared with the corresponding value from human stomach.

stomach and small intestine. The measured complex permittivities for pig stomach in Fig. 8 and small intestine in Fig. 9 are smaller than those obtained from the corresponding human organs. The texture and contents of these organs are considered to be the cause of these differences.

The lung, liver, heart, and kidney have regular texture and contents. Blood is also a consistent fluid. However, stomach and small intestine can be filled with a range of arbitrary materials such as air, intestinal juices, food, etc. because two organs are the parts of the digestive tract. During our experiment, the pig stomach is mostly filled with air as illustrated in Fig. 10(a), while the pig small intestine is filled with arbitrary materials as shown in Fig. 10(b). Consequently, the converted complex permittivities of the stomach and small intestine are relatively smaller than the corresponding values from human organs. 


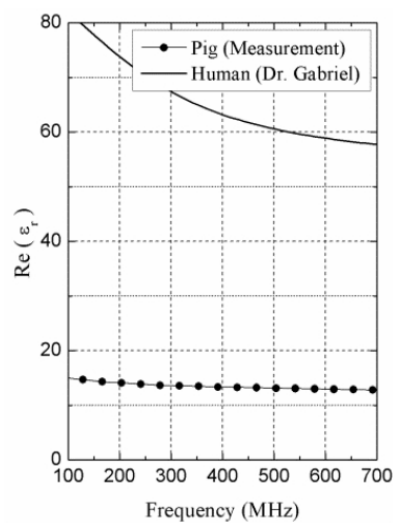

(a) Real part

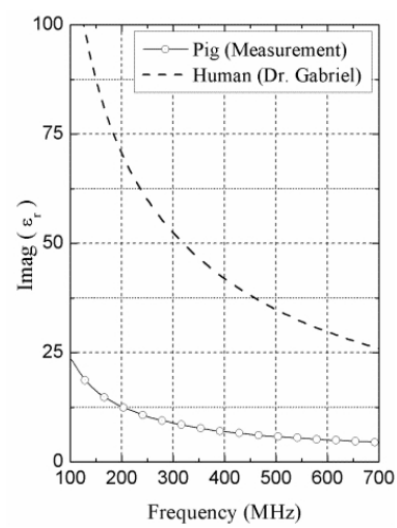

(b) Imaginary part
Fig. 9. Measured electrical property of pig small intestine compared with the corresponding value from human small intestine.

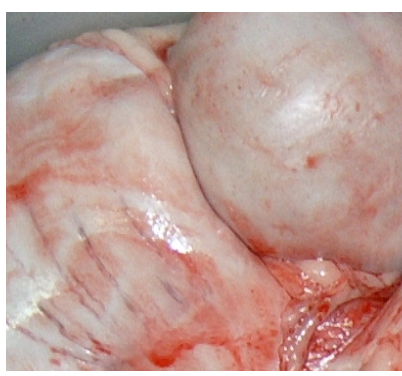

(a) Stomach filled with air

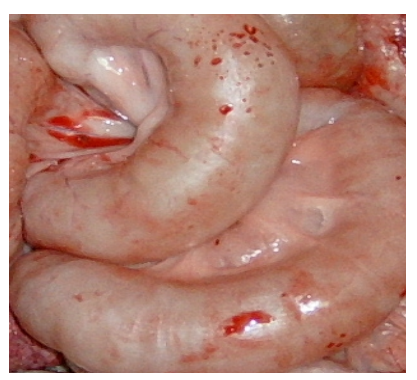

(b) Small intestine filled with arbitrary materials
Fig. 10. Some internal organs of pig employed during measurement in vitro.

Based on the results of this comparison, pig internal organs can be classified into three groups: organs with regular texture and contents, regular fluid, and digestive tract. Organs that have regular texture and contents (lung, liver, heart, and kidney) or regular fluids (blood) assure similar electrical properties with human organs. Therefore, a human phantom can be developed using these organs from a pig. However, careful attention is required for utilizing pig digestive tract such as stomach, small intestine, etc. because the complex permittivities of these organs are affected by the materials filling those organs.

\section{Conclusion}

Complex permittivities of pig internal organs were measured at as laughter house using an open-ended coaxial probe and an improved virtual transmission-line model. The measured results were compared with the corresponding values of human organs to confirm the similarity between these values. The compared results of lung, liver, heart, and kidney composed of regular texture and contents were close to the reported values for human organs. The complex permittivities of pig blood were also similar to reported human values. In contrast, the values for stomach and small intestine showed large deviations according to the material filling those organs. It leads us to conclude that pig organs that have regular texture and contents are suitable for developing a phantom. In contrast, some caution is needed when pig digestive tract are considered for use in a phantom.

\section{References}

[1] W. G. Scanlon, J. B. Burns, and N. E. Evans, "Radiowave propagation from a tissue-implanted source at $418 \mathrm{MHz}$ and $916.5 \mathrm{MHz}, "$ IEEE Trans. Biomed. Eng., vol. 47, pp. 527-534, Apr. 2000.

[2] L. C. Chirwa, P. A. Hammond, S. Roy, and D. R. S. Cumming, "Electromagnetic radiation from ingested sources in the human intestine between 150 $\mathrm{MHz}$ and $1.2 \mathrm{GHz}, "$ IEEE Trans. Biomed. Eng., vol. 50, pp. 484-492, Apr. 2003.

[3] Y. Chan, M. Q. -H. Meng, K. -L. Wu, and X. Wang, "Experimental study of radiation efficiency from an ingested source inside a human body model," in Proc. 27th IEEE Engineering in Medicine and Biology, Shanghai, pp. 7754-7757, 2005.

[4] J.-H. Jung, S.-W. Kim, Y.-S. Kim, and S.-Y. Kim, "Electromagnetic propagation from the intestine-ingested source in the human body model," IEEE Trans. Antennas Propag., vol. 58, no. 4, pp. 16831688, May 2010.

[5] J.-H. Jung, S.-Y. Kim, "The effect of an ingested source's location in a human body model on electromagnetic propagation," JKIEES, vol. 11, no. 1, pp. 51-55, 2011.

[6] C. Gabriel, S. Gabriel, and E. Corthout, "The dielectric properties or biological tissues: I. Literature survey," Phys. Med. Biol., vol. 41, pp. 2231-2249, 1996.

[7] H. Shin, S.-Y. Hyun, S.-W. Kim, and S.-Y. Kim, "An efficient model of an open-ended coaxial-line probe for measuring the complex permittivity," in Proc. SPIE, in Subsurface Sensing Technology and Application II, vol. 4129, pp. 332-339, Jul.-Aug., 2000.

[8] S. Gabriel, R. W. Lau, and C. Gabriel, "The dielectric properties of biological tissues: II. Measurements in the frequency range $10 \mathrm{~Hz}$ to $20 \mathrm{GHz}$," Phys. Med. Biol., vol. 41, pp. 2251-2269, 1996. 
Ji-Hyun Jung

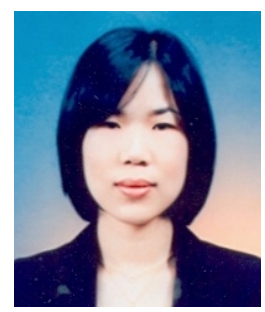

received the B.S. degree in electrical engineering from the Dankook University, Seoul, Korea, in 2004 and the M.S. and $\mathrm{Ph} . \mathrm{D}$. degrees in radio sciences and engineering from the Korea University, Seoul, Korea, in 2006 and 2011, respectively.

From 2004 to 2011, she was a Student Researcher in the Korea Institute of Science and Technology, Seoul, Korea. She is currently a Postdoctoral Research Fellow at the Korea Institute of Science and Technology. Her research interests include finite-difference time-domain analysis, electromagnetic propagation through the human body model, small antenna development for biomedical devices, and dielectric constant measurement.

\section{Se-Yun Kim}

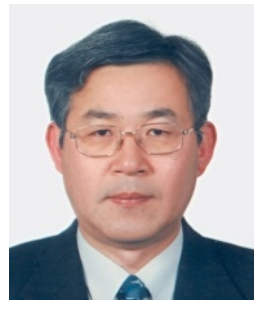

received the B.S. degree from the Seoul National University, Seoul, Korea, in 1978 and the M.S. and Ph.D. degrees from the Korea Advanced Institute of Science and Technology, Seoul, Korea, in 1980 and 1984, respectively, all in electrical engineering.

He was a Postdoctoral Research Fellow from 1984 to 1986 at the Korea Advanced Institute of Science and Technology. In 1986, he joined the System Technology Division at the Korea Institute of Science and Technology, Seoul, Korea, as a Senior Researcher, and where he is currently a Principal Researcher. His research interests include electromagnetic diffraction, microwave imaging, and geophysical probing.

Dr. Kim received the Order of National Security Merit from the Korean Government in 1990. 